\title{
Acceptability of the Dapivirine Vaginal Ring for HIV-1 Prevention and Association with Adherence in a Phase III Trial
}

\author{
Ashley J. Mayo ${ }^{1}$ (D) - Erica N. Browne ${ }^{2}$ - Elizabeth T. Montgomery ${ }^{2} \cdot$ Kristine Torjesen $^{1} \cdot$ Thesla Palanee-Phillips $^{3}$. \\ Nitesha Jeenarain ${ }^{4} \cdot$ Linly Seyama $^{5} \cdot$ Kubashni Woeber $^{4} \cdot$ Ishana Harkoo $^{6} \cdot$ Krishnaveni Reddy $^{3} \cdot$ Tchangani Tembo $^{7}$. \\ Prisca Mutero ${ }^{8} \cdot$ Thelma Tauya $^{8} \cdot$ Miria Chitukuta ${ }^{8} \cdot$ Brenda Gati Mirembe $^{9} \cdot$ Lydia Soto-Torres $^{10}$. \\ Elizabeth R. Brown ${ }^{11}$ • Jared M. Baeten ${ }^{12,14}$. Ariane van der Straten ${ }^{2,13}$. for the MTN-020/ASPIRE study team
}

Accepted: 17 February 2021 / Published online: 13 March 2021

(c) The Author(s) 2021

\begin{abstract}
We evaluated the acceptability of the $25 \mathrm{mg}$ dapivirine vaginal ring (DVR) as an HIV prevention intervention and its influence on DVR adherence in the MTN-020/ASPIRE phase III trial. Acceptability measures were captured using ACASI at month 3 and end of product use (median 24 months, IQR 15-30). Monthly returned rings were classified as nonadherent if dapivirine release rate was $\leq 0.9 \mathrm{mg} / \mathrm{month}$. Associations between acceptability measures and nonadherence were estimated using Poisson regression models with robust standard errors. At month $3(\mathrm{~N}=2334), 88 \%$ reported DVR was comfortable, $80 \%$ were unaware of it during daily activities, and $74 \%$ never felt it during sex. At exit, $66 \%$ were 'very likely' to use DVR in the future. Acceptability was found to differ significantly by country across several measures including wearing the ring during sex, during menses, partner acceptability, impact on sexual pleasure and willingness to use the ring in the future. Risk of nonadherence at month 12 was elevated if DVR was felt during sex at month 3 (aRR 1.67, 95\% CI 1.26, 2.23). Risk of nonadherence in the last year of study participation was elevated if, at exit, participants minded wearing during sex (aRR 2.08, 95\% CI 1.52, 2.85), during menses (aRR 1.57, 95\% CI 1.06, 2.32), reported a problematic change to the vaginal environment (aRR 1.57, 95\% CI 1.12, 2.21), and were not "very likely" to use DVR in the future (aRR 1.31, 95\% CI 1.02, 1.68). DVR acceptability was overall high yet varied by country. Addressing perceived ring interference with sex, menses, or problematic changes to the vaginal environment in future interventions could help improve adherence, as could embracing sex-positive messaging related to ring use and increased pleasure.
\end{abstract}

Trial Registration ClinicalTrials.gov Identifier: NCT01617096.

Keywords HIV prevention $\cdot$ Acceptability $\cdot$ Adherence $\cdot$ Vaginal ring $\cdot$ Sub-Saharan Africa

Collaborators of the MTN-020/ASPIRE Study Team are listed in

"Acknowledgements".

Ashley J. Mayo

amayo@ fhi360.org

FHI 360, Durham, NC, USA

2 Women's Global Health Imperative, RTI International, Berkeley, CA, USA

3 Wits Reproductive Health and HIV Institute, University of the Witwatersrand, Johannesburg, South Africa

4 HIV Prevention Research Unit, South African Medical Research Council, Durban, South Africa

5 College of Medicine-Johns Hopkins Research Project, Queen Elizabeth Central Hospital, Blantyre, Malawi

6 Centre for AIDS Programme of Research in South Africa, University of KwaZulu-Natal, Durban, South Africa
University of North Carolina Project, Lilongwe, Malawi

8 University of Zimbabwe Clinical Trials Research Centre (UZ-CTRC), Harare, Zimbabwe

9 Makerere University - Johns Hopkins University (MU-JHU) Research Collaboration CRS, Kampala, Uganda

10 National Institute of Allergy and Infectious Diseases, National Institutes of Health, Bethesda, MD, USA

11 Fred Hutchinson Cancer Research Center, Seattle, WA, USA

12 University of Washington, Seattle, WA, USA

13 University of California San Francisco (UCSF), San Francisco, CA, USA

14 Present Address: Gilead Sciences, Foster City, CA, USA 


\section{Introduction}

More than half of people living with HIV worldwide are women and those living in sub-Saharan Africa bear a disproportionate burden of new HIV infections each year [1]. They require and desire options for HIV prevention that are safe, effective, and acceptable $[2,3]$. The dapivirine vaginal ring (DVR) is one potential option. Two randomized, placebo controlled, phase III trials in healthy sexually-active women, MTN-020/ASPIRE and IPM-027/ The Ring Study, demonstrated the monthly DVR was welltolerated and reduced HIV incidence [4, 5]. In ASPIRE, higher risk reduction was estimated with measures indicating higher adherence [6]. In earlier phase I and II trials the DVR was found to be highly acceptable [7-10].

Assessment of product acceptability is not well standardized and is too often measured as a singular concept. As described by Mensch et al. [11] a framework that evaluates components of acceptability-including dosing regimen, product use attributes, effect of the product on sex, and partner's attitudes-may help better define the influence of product acceptability on adherence of new HIV prevention methods.

In previous trials, acceptability of vaginal rings was high across several measures, including overall willingness to use in the future (if found effective for HIV prevention) [7, 9], ease of insertion/removal [7, 9, 10], feeling comfortable $[7,9,10]$, and being unnoticeable during daily activities or once inserted $[7,9,10]$. Furthermore, early trials indicate most do not feel the ring during sex [7] or that it does not interfere with sex [9]. Per participant report, their male partners were more likely to have felt the ring during sex, however, only a minority reported this to be a problem for continued ring use [7]. Qualitative analysis from ASPIRE indicated that participants generally liked the ring better with experience, that adherence challenges could usually be overcome with staff or peer support, and that male partners were a commonly cited influence on ring acceptability and adherence [12]. The ring also impacted sexual experience in many ways, for some positively and for others negatively [13].

Here we present a quantitative analysis of DVR acceptability in the ASPIRE trial, one of the first cohorts to use the ring for an extended period (up to 3 years). We evaluated dimensions of acceptability, changes throughout the trial, and their independent influence on an objective measure of adherence. Understanding the acceptability of the DVR, the importance of each component, and the potential influence of acceptability on product adherence, is critical for supporting the ring's successful roll-out and use in sub-Saharan Africa.

\section{Methods}

\section{Study Population and Design}

MTN-020/ASPIRE (NCT01617096) was a phase III randomized, double-blind, placebo-controlled clinical trial conducted across 15 sites in Malawi (two sites), South Africa (nine sites), Uganda (one site), and Zimbabwe (three sites) that enrolled 2629 women aged 18-45 between August 2012 and June 2015. Participants were randomized in a 1:1 ratio to use either a monthly silicone elastomer vaginal matrix ring containing $25 \mathrm{mg}$ of dapivirine or a placebo ring. Participants were counseled on how to insert and remove the ring and were instructed to keep the ring inserted for the entire month. Eight months after trial initiation, several site-specific participant engagement activities and monitoring strategies were implemented to improve adherence and retention in the trial [14]. Study visits occurred monthly, and at each visit, participants received HIV-1 serologic and pregnancy testing, safety monitoring, and individualized adherence counseling based on reported ring use experiences. Participants were followed for a minimum of one year and for a maximum of 33 months. Intention-to-treat analysis showed 27\% reduction in HIV incidence [95\% confidence interval $(\mathrm{CI}), 1-46 ; \mathrm{P}=0.05$ ] in the DVR arm compared to placebo. Additional details of the trial design, recruitment, and results have been published previously [4].

\section{Measures}

\section{Acceptability}

Acceptability data were captured using audio computerassisted self-interview (ACASI) at the month-3 visit and at the Product Use End visit (PUEV), the penultimate visit when study product dispensing was permanently discontinued, among participants who self-reported using the ring. Participants were asked during each ACASI to reflect on their experiences using the ring in the prior 3 months. In addition, the PUEV ACASI collected participants' views of the ring in general, based on their experience throughout the trial. Participants also completed an ACASI at baseline which assessed sexual behavioral characteristics and likelihood of future ring use. Acceptability measures, response options, and frequency of assessment during the trial are summarized in Table 1.

\section{Adherence}

For this analysis, adherence was determined by residual dapivirine in returned vaginal rings, which were collected 
Table 1 ASPIRE acceptability measures

\begin{tabular}{|c|c|c|c|c|}
\hline \multirow[t]{2}{*}{ Acceptability measure $^{\mathrm{a}}$} & \multirow[t]{2}{*}{ Response options } & \multicolumn{3}{|c|}{ Assessed at visit } \\
\hline & & Enrollment & Month 3 & PUEV $^{b}$ \\
\hline \multicolumn{5}{|l|}{ Overall acceptability } \\
\hline $\begin{array}{l}\text { If in the future a vaginal ring was available that } \\
\text { provided some protection against HIV, and it was } \\
\text { similar to the one you used in this study, how likely } \\
\text { would you be to keep it inserted in your vagina } \\
\text { every day? }\end{array}$ & Very unlikely, unlikely, likely, very likely & $\mathrm{X}$ & & $\mathrm{X}$ \\
\hline \multicolumn{5}{|l|}{ Use attributes } \\
\hline $\begin{array}{l}\text { How difficult was it to insert the vaginal ring the last } \\
\text { time you inserted it? }\end{array}$ & Very difficult, somewhat difficult, not difficult at all & & $\mathrm{X}$ & $\mathrm{X}$ \\
\hline $\begin{array}{l}\text { How difficult was it to take the vaginal ring out the } \\
\text { last time you took it out? }\end{array}$ & Very difficult, somewhat difficult, not difficult at all & & $\mathrm{X}$ & $\mathrm{X}$ \\
\hline $\begin{array}{l}\text { In the past } 3 \text { months, how did it feel to have the vagi- } \\
\text { nal ring inside you every day? }\end{array}$ & $\begin{array}{l}\text { Usually comfortable, sometimes uncomfortable, usu- } \\
\text { ally uncomfortable }\end{array}$ & & $\mathrm{X}$ & $\mathrm{X}$ \\
\hline $\begin{array}{l}\text { In the past } 3 \text { months, were you aware of the vaginal } \\
\text { ring during your normal daily activities? }\end{array}$ & Most of the time, sometimes, never & & $\mathrm{X}$ & $\mathrm{X}$ \\
\hline Did you mind wearing the ring during menses? & $\begin{array}{l}\text { Yes, no, did not wear the vaginal ring during menses, } \\
\text { did not have menses during the study }\end{array}$ & & & $\mathrm{X}$ \\
\hline $\begin{array}{l}\text { Have you noticed any of the following changes in } \\
\text { your vagina while wearing the vaginal ring: vagina } \\
\text { was wetter, vaginas was drier? }\end{array}$ & $\begin{array}{l}\text { Vagina was wetter, vagina was drier, no change } \\
\text { noticed }\end{array}$ & & & $\mathrm{X}$ \\
\hline $\begin{array}{l}\text { [If wetter or drier]: was this change a problem for } \\
\text { you? }\end{array}$ & Yes, no & & & \\
\hline \multicolumn{5}{|l|}{ Effects on sex } \\
\hline $\begin{array}{l}\text { In the past } 3 \text { months, how often did you feel the } \\
\text { vaginal ring inside you when you had sex? }\end{array}$ & $\begin{array}{l}\text { Most of the time, sometimes, never, did not have sex } \\
\text { in the past } 3 \text { months }\end{array}$ & & $\mathrm{X}$ & $\mathrm{X}$ \\
\hline $\begin{array}{l}\text { In the past } 3 \text { months, did any of your partners feel the } \\
\text { vaginal ring inside of you when you had sex? }\end{array}$ & Yes, no, don't know & & $\mathrm{X}$ & $\mathrm{X}$ \\
\hline $\begin{array}{l}\text { How does the vaginal ring affect your sexual pleas- } \\
\text { ure? }\end{array}$ & $\begin{array}{l}\text { Increases pleasure, does not change pleasure, } \\
\text { decreases pleasure }\end{array}$ & & & $\mathrm{X}$ \\
\hline Did you mind wearing the ring during sex? & $\begin{array}{l}\text { Yes, no, did not wear the vaginal ring during sex, did } \\
\text { not have sex during the study }\end{array}$ & & & $\mathrm{X}$ \\
\hline \multicolumn{5}{|l|}{ Partner's attitude } \\
\hline $\begin{array}{l}\text { Was the vaginal ring acceptable to your primary } \\
\text { partner? }\end{array}$ & Yes, no, don't know & & & $\mathrm{X}$ \\
\hline $\begin{array}{l}\text { Has your primary sex partner ever asked you to stop } \\
\text { wearing the ring? }\end{array}$ & Yes, no & & & $\mathrm{X}$ \\
\hline
\end{tabular}

${ }^{a}$ Measures are organized by component of acceptability as outlined by Mensch et al. [11]

${ }^{\mathrm{b}} P U E V$ product use end visit, the penultimate visit when study product dispensing was permanently discontinued (median 24 months, IQR 15-30 months)

monthly starting approximately one calendar year after trial initiation. Acetone extraction and high-pressure liquid chromatography measured residual dapivirine in returned used rings. Previous research has established that with consistent use of the ring in a 28-day period, at least $4 \mathrm{mg}$ of dapivirine is released [15]. From lab measures of unused rings, dapivirine release rates $\leq 0.9 \mathrm{mg}$ per month indicated no or very low use of the ring (the equivalent of one standard deviation of lab measurement error). Because study visits did not occur exactly every 28 days, adherence was based on the ratio of the amount of dapivirine released $(25 \mathrm{mg}$ minus the remaining dapivirine) to the number of days since the ring was dispensed. Rings with a release rate equivalent to $\leq 0.9 \mathrm{mg}$ per month were classified as nonadherent [16]. If a participant indicated a ring was lost or did not returned a ring, the ring was assumed to have not been used (i.e., release rate $\leq 0.9 \mathrm{mg} / \mathrm{month}$ ).

\section{Analysis}

Participants who completed acceptability measures during at least one follow-up ACASI (month 3, PUEV) were 
included in this analysis. Descriptive characteristics of the analysis sample are presented by country to highlight differences in the study population. Chi-square tests or $t$ tests were used to evaluate the differences observed. We used logistic regression models, adjusted for country to assess differences in sociodemographic characteristics between the total enrolled sample and the analytic sample. Acceptability measures were summarized by study visit to evaluate changes over time and by country, as per above. Based on the response distribution and possible positive response bias for the overall acceptability measure, analyses compared "very likely" future use to less than very likely (i.e. "likely", "unlikely," or "very unlikely"). To test for differences in acceptability over time, we used a mixed effects logistic regression model for each acceptability measure. Models including fixed effects for country, treatment arm, and total months of follow-up and a random effect for participant. For measures only assessed at PUEV, Chi-square tests were used to compare responses by country. Given younger participants were found to be less adherent in the trial [4], we used logistic regression models to explore if acceptability differed between younger and older participants, adjusted for country, treatment arm, and months of follow-up. Young participants were defined as those age 18-21 years at enrollment.

The adherence portion of the analysis excluded all participants randomized to the placebo ring, given adherence was determined by dapivirine drug concentrations. We estimated how early acceptability of the ring (from the month-3 ACASI) influenced nonadherence at month-12, since most participants had residual dapivirine measures at this visit. We also assessed the relationship between acceptability measures collected only at PUEV and nonadherence within the last year of study participation. Nonadherence was dichotomized and defined by having three nonadherent rings in the last 12 months. Only those with at least 5 rings dispensed in the last year were included. Most (80\%) had 12 rings dispensed in their last year in the study; $9 \%$ had $<5$ rings. As a sensitivity analysis, we refit models only with those who had 12 rings dispensed in the last 12 months and found similar effects. We estimated separate Poisson regression models with robust standard errors for each acceptability measure to determine if it was associated with nonadherence. Models were stratified by age for acceptability measures found to differ by age group. All models controlled for country, months of follow-up, and enrollment post initiation of the adherence monitoring and engagement activities.

All analyses were performed using Stata 15.0 (StataCorp LLC, College Station, TX). P values $<0.05$ were considered significant. Local ethical approval was obtained from all study sites prior to trial implementation. Informed consent was obtained from all individual participants included in the study [4].

\section{Results}

Among the 2629 participants enrolled in the ASPIRE trial, $2562(97 \%)$ completed the acceptability measures on at least one follow-up ACASI and were included in the analysis. There were no significant differences between participants included in the analytic sample and those without ACASI who were excluded (all $\mathrm{P} \geq 0.09$ ). Descriptive characteristics of the analytic sample are presented in Table 2. Participants across countries differed on almost every characteristic evaluated (Supplemental Table 1). Across all sites, the average age of participants was 27 with $20 \%$ considered young (between the ages of 18-21); $46 \%$ had completed secondary school, $41 \%$ were married, and $45 \%$ earned an income of their own. Nearly all $(98 \%)$ had a primary partner and were parous (92\%). At enrollment, 58\% had more than one male sex partner in the past 3 months. On average, participants were followed for 21.5 months (median 21, interquartile range 14-29 months).

Table 2 Baseline characteristics of participants in the ASPIRE trial (Aug 2012-Jun 2015) who completed acceptability measures at month-3 and/or PUEV

\begin{tabular}{|c|c|c|}
\hline & \multicolumn{2}{|l|}{ Total } \\
\hline & $\mathrm{N}$ & $(\%)$ \\
\hline Total & 2562 & $(100)$ \\
\hline Age, years-mean, median (IQR) & $27.2,26$ & $(22-31)$ \\
\hline $18-21$ & 507 & $(20)$ \\
\hline $22-45$ & 2055 & $(80)$ \\
\hline Completed secondary school & 1172 & $(46)$ \\
\hline Earns own income & 1156 & $(45)$ \\
\hline Has primary sex partner & 2496 & $(98)$ \\
\hline Currently married & 1058 & $(41)$ \\
\hline$\geq 2$ male sex partners in past 3 mo & 1489 & $(58)$ \\
\hline Transactional sex in past year & 156 & (6) \\
\hline Parity $>0$ & 2350 & $(92)$ \\
\hline \multicolumn{3}{|l|}{ Current contraceptive method } \\
\hline Injectable & 1407 & $(55)$ \\
\hline Implant & 494 & (19) \\
\hline Intrauterine device (IUD) & 319 & (13) \\
\hline Oral contraceptive pills & 278 & $(11)$ \\
\hline Male condoms & 94 & (4) \\
\hline Sterilization $^{\mathrm{a}}$ & 77 & (3) \\
\hline
\end{tabular}

$P U E V$ product use end visit, $I Q R$ interquartile range

${ }^{\text {a }}$ Tubal ligation/hysterectomy/laparoscopy/other surgical procedure that causes sterilization 


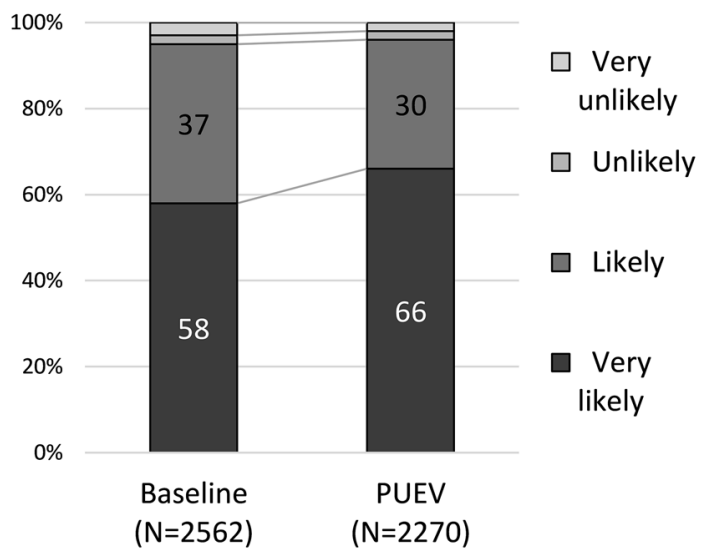

Fig. 1 Overall acceptability of the dapivirine vaginal ring, as measured by future likelihood of use at baseline and PUEV

\section{Acceptability}

Overall, at PUEV nearly all participants reported they were "very likely" (66\%) or "likely" (30\%) to use a monthly vaginal ring in the future. More were "very likely" compared to baseline [adjusted odds ratio (AOR): 1.5 95\% CI $1.3,1.7, \mathrm{P}<0.001$; Fig. 1). There were significant differences in future willingness to use by country at both baseline and PUEV, with about half of participants in Malawi and South Africa stating they were "very likely" compared to about three-quarters in Uganda and Zimbabwe (Supplemental Table 1, Table 4).

Acceptability of the ring (based on prior 3 months of use) collected at month 3 and PUEV are compared in Table 3. Much of the analytic sample had month 3 ACASI data available $(\mathrm{n}=2474,97 \%)$, and among these, 2334 (94\%) self-reported using the ring in the past 3 months and provided responses to acceptability measures. In general, the ring was found acceptable across several components of acceptability. Most participants found the ring not difficult to insert $(85 \%)$ or remove $(76 \%)$, usually comfortable $(88 \%)$, and were unaware of it during normal daily activities $(80 \%)$ or during sex $(74 \%)$. Sixty-nine percent reported that their partner(s) did not feel the ring during sex and $12 \%$ did not know if their partner(s) had felt the ring during sex in the past 3 months.

Although 2469 participants completed their PUEV visit for ASPIRE, ACASI data were available for 2270 participants $(92 \%)$, with missing data largely attributable to transmission errors primarily at two sites. Of the 2270 participants who completed the PUEV ACASI, 2075 (91\%) stated they had used the ring within the past 3 months and provided responses to acceptability measures. Compared to month 3 , a greater proportion of participants at PUEV found the ring not difficult to insert (93\%), usually comfortable (92\%), and were unaware of it during normal activities $(84 \%)$.
Table 3 Acceptability of the dapivirine vaginal ring based on use in the past 3 months, at month-3 visit and PUEV

\begin{tabular}{|c|c|c|c|c|}
\hline \multirow[b]{3}{*}{ Total who used ring in past 3 months ${ }^{\mathrm{a}}$} & \multicolumn{2}{|c|}{ Month 3} & \multicolumn{2}{|l|}{ PUEV } \\
\hline & $\mathrm{N}$ & $(\%)$ & $\mathrm{N}$ & $(\%)$ \\
\hline & 2334 & $(100)$ & 2075 & $(100)$ \\
\hline \multicolumn{5}{|l|}{ Use attributes: ease and comfort of use } \\
\hline \multicolumn{5}{|l|}{ How difficult was it to insert the ring $?^{b}$} \\
\hline Very/somewhat difficult & 346 & (15) & 128 & (6) \\
\hline Not difficult at all & 1974 & $(85)$ & 1938 & (93) \\
\hline \multicolumn{5}{|l|}{ How difficult was it to take the ring out? ${ }^{\mathrm{b}}$} \\
\hline Very/somewhat difficult & 326 & $(14)$ & 197 & $(10)$ \\
\hline Not difficult at all & 1776 & $(76)$ & 1617 & $(78)$ \\
\hline Never took out ring in past 3 months & 232 & $(10)$ & 261 & $(13)$ \\
\hline \multicolumn{5}{|l|}{ Use attributes: physical sensation in situ } \\
\hline \multicolumn{5}{|c|}{ How did it feel to have the ring inside you every day ${ }^{\mathrm{b}}$} \\
\hline Usually comfortable & 2047 & $(88)$ & 1907 & $(92)$ \\
\hline Sometimes/usually uncomfortable & 286 & (12) & 167 & (8) \\
\hline \multicolumn{5}{|c|}{ Were you aware of the ring during normal daily activities? ${ }^{\mathrm{b}}$} \\
\hline Most/some of the time & 478 & $(21)$ & 342 & $(16)$ \\
\hline Never & 1855 & $(80)$ & 1732 & $(84)$ \\
\hline \multicolumn{5}{|l|}{ Effects on sex } \\
\hline Total who had sex in past 3 months & 2310 & $(99)$ & 2011 & $(97)$ \\
\hline \multicolumn{5}{|c|}{ How often did you feel the ring during sex? } \\
\hline Most/some of the time & 592 & $(26)$ & 474 & $(24)$ \\
\hline Never & 1716 & $(74)$ & 1537 & $(76)$ \\
\hline \multicolumn{5}{|c|}{ Did any of your partners feel the ring during sex ${ }^{\text {b }}$} \\
\hline Yes & 444 & $(19)$ & 348 & $(17)$ \\
\hline No & 1600 & $(69)$ & 1389 & $(69)$ \\
\hline Don’t know & 265 & (12) & 273 & (14) \\
\hline
\end{tabular}

PUEV product use end visit

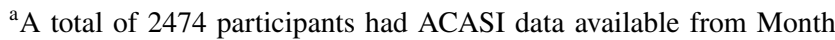
3, and 2270 from PUEV. PUEV ACASI data were missing from 2 sites due to transmission errors

${ }^{\mathrm{b}}$ Significant difference between Month-3 and PUEV ( $\left.\mathrm{p}<0.05\right)$. Differences over time were tested using mixed-effect regression models, controlling for country, treatment arm, and number of months of follow-up

In addition, at PUEV, participants were asked about their general opinions of the vaginal ring over the entire study period. Acceptability was found to differ significantly by country (Table 4). Significantly more participants in Malawi (35\%) and Uganda (85\%) minded wearing the ring during sex compared to South Africans (10\%) and Zimbabweans $(<1 \%)$. Similarly, more in Malawi $(35 \%)$ and Uganda (77\%) minded wearing the ring during menses than in South Africa (8\%) and Zimbabwe (1\%). Overall, $75 \%$ of those who minded wearing the ring during sex also minded wearing it during menses. Thirty percent $(n=683)$ noticed a change in the vaginal environment while wearing the ring (either the vagina was wetter $(20 \%)$ or drier $(10 \%)) ; 187(8 \%)$ reported this change as problematic. Most participants (58\%) reported 


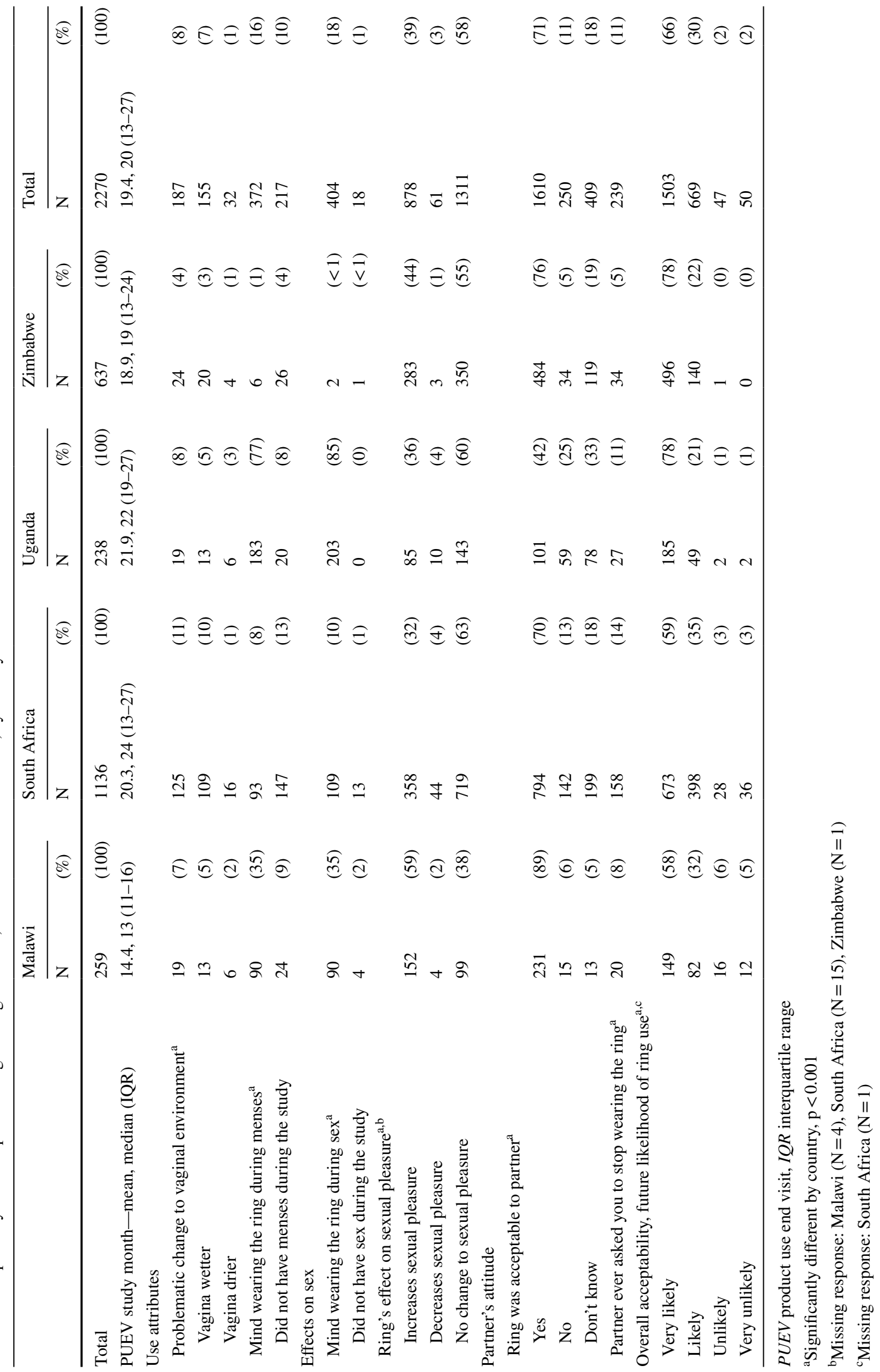


the ring did not affect sexual pleasure, while $39 \%$ stated it increased pleasure. Overall, the majority (71\%) said the ring was acceptable to their partner, except in Uganda, where less than half (42\%) reported the ring was acceptable to their partner.

Most acceptability measures were similar between younger and older participants apart from their perceptions of their partner's acceptability; those who were younger were more likely to state the ring was not acceptable to their primary partner (16\% vs $10 \%$, AOR $1.9,95 \%$ CI $1.4,2.7$; $\mathrm{P}<0.001)$.

\section{Acceptability and Nonadherence}

Table 5 presents the associations between acceptability and nonadherence among participants who were randomized to use the dapivirine ring. First, we estimated how initial acceptability of the ring, measured at month 3 , influenced nonadherence at Month $12(\mathrm{~N}=1058)$. Sixteen-percent of participants were deemed nonadherent based on the ring from month 12 having little to no evidence of use. Participants were more likely to be nonadherent if they felt the ring during sex in the first three months [adjusted relative risk (aRR) 1.67, 95\% CI 1.26, 2.23; P<0.001] and if they said they had a partner who felt the ring during sex (aRR 1.39, 95\% CI 0.99, 1.95; $\mathrm{P}=0.06$ ). Participants who were aware of the ring during normal activities in the first 3 months seemed modestly more likely to be nonadherent although these results were inconclusive (aRR $1.24,95 \%$ CI 0.91 , $1.69 ; \mathrm{P}=0.17)$. Initial difficulty with insertion and experiencing discomfort with wearing the ring during the first three months were not associated with nonadherence.

Table 5 Associations between acceptability and nonadherence among women randomized to dapivirine vaginal ring

\begin{tabular}{|c|c|c|c|c|}
\hline & \multirow[t]{2}{*}{$\mathrm{N}(\%)$} & \multicolumn{3}{|c|}{ Nonadherence, at Month $12^{\mathrm{a}}$} \\
\hline & & $\mathrm{aRR}^{\mathrm{b}}$ & $95 \% \mathrm{CI}$ & $\mathrm{p}$ value \\
\hline \multicolumn{5}{|l|}{ Acceptability measures from month-3 ACASI $(\mathrm{N}=1058)$} \\
\hline \multicolumn{5}{|l|}{ Use attributes } \\
\hline Sometimes/usually uncomfortable to have ring inside every day & $136(13)$ & 1.07 & $(0.73,1.56)$ & 0.72 \\
\hline Very/somewhat difficult to insert & $150(14)$ & 1.01 & $(0.70,1.46)$ & 0.95 \\
\hline Some/most of the time aware of ring during normal activities & $216(20)$ & 1.24 & $(0.91,1.69)$ & 0.17 \\
\hline \multicolumn{5}{|l|}{ Effects on sex } \\
\hline Felt ring during sex & $256(24)$ & 1.67 & $(1.26,2.23)$ & $<0.001$ \\
\hline \multirow[t]{3}{*}{ Partner felt ring during sex (yes vs. no) } & $192(18)$ & 1.39 & $(0.99,1.95)$ & 0.06 \\
\hline & $\mathrm{N}(\%)$ & \multicolumn{3}{|c|}{ Nonadherence, in last year of study ${ }^{\mathrm{c}}$} \\
\hline & & $\mathrm{aRR}^{\mathrm{b}}$ & $95 \% \mathrm{CI}$ & $\mathrm{p}$ value \\
\hline \multicolumn{5}{|l|}{ Acceptability measures from PUEV ACASI $(\mathrm{N}=1042)$} \\
\hline \multicolumn{5}{|l|}{ Use attributes } \\
\hline Problematic change to vaginal environment & $87(8)$ & 1.57 & $(1.12,2.21)$ & 0.009 \\
\hline Mind wearing during menses & $158(15)$ & 1.57 & $(1.06,2.32)$ & 0.02 \\
\hline \multicolumn{5}{|l|}{ Effects on sex } \\
\hline Mind wearing during sex & $178(17)$ & 2.08 & $(1.52,2.85)$ & $<0.001$ \\
\hline \multicolumn{5}{|l|}{ Ring's effect on sexual pleasure ${ }^{\mathrm{d}}$} \\
\hline Increases sexual pleasure & $430(41)$ & Ref & - & - \\
\hline No change & $582(56)$ & 1.40 & $(1.07,1.83)$ & 0.01 \\
\hline Decreases sexual pleasure & $21(2)$ & 1.68 & $(0.82,3.45)$ & 0.16 \\
\hline \multicolumn{5}{|l|}{ Partner's attitude } \\
\hline Ring acceptable to partner (no vs yes) & $104(10)$ & 1.38 & $(0.98,1.96)$ & 0.07 \\
\hline \multicolumn{5}{|l|}{ General acceptability } \\
\hline Less than "very likely" to use in the future & $345(33)$ & 1.31 & $(1.02,1.68)$ & 0.03 \\
\hline
\end{tabular}

ACASI audio computer-assisted self-interview, $C I$ confidence interval, $P U E V$ product use end visit

${ }^{\mathrm{a}}$ Month 12 ring had dapivirine release rate $\leq 0.9 \mathrm{mg} / \mathrm{month}(\mathrm{N}=165$ of $1058 ; 16 \%)$

${ }^{\mathrm{b}}$ Adjusted relative risk (aRR) estimated using separate Poisson regression models with robust standard errors for each acceptability measure. All models adjusted for country, total months of enrollment, and enrollment post adherence intervention initiation

${ }^{\mathrm{c}}$ Three rings in last year of study had dapivirine release rate $\leq 0.9 \mathrm{mg} / \mathrm{month}(\mathrm{N}=200$ of $1042 ; 19 \%)$

${ }^{\mathrm{d}}$ Missing response $(\mathrm{N}=9)$ 
Second, we assessed how additional acceptability measures collected at PUEV were associated with nonadherence in the last year of the study $(\mathrm{N}=1042) ; 19 \%$ met the definition of nonadherent. Participants who stated they minded wearing the ring during sex were twice as likely to be nonadherent (aRR 2.08, 95\% CI 1.52, 2.85; P<0.001). Those who reported a problematic change to the vaginal environment (aRR 1.57, 95\% CI 1.12, 2.21; $\mathrm{P}=0.009)$, who minded wearing the ring during menses (aRR 1.57, 95\% CI 1.06, 2.32; $\mathrm{P}=0.02$ ), and who were less than "very likely" to use a vaginal ring in the future (aRR 1.31, 95\% CI 1.02, 1.68; $\mathrm{P}=0.03$ ) also had elevated risk of nonadherence. Risk of nonadherence was also associated with impact on sexual pleasure; those who reported no change (aRR 1.40, 95\% CI $1.07,1.83 ; \mathrm{P}=0.01$ ) or a decrease in pleasure (aRR 1.68 , $95 \%$ CI $0.82,3.45$ ) were more likely to be nonadherent than those who found the ring increased sexual pleasure. Participants who reported that the ring was not acceptable to their partners were somewhat more likely to be nonadherent, although these results were inconclusive (aRR 1.38, 95\% CI $0.98,1.96)$. As partner acceptability was the only measure found to be different by age, we explored the impact of partner acceptability on nonadherence stratified by age. Those aged 22-45 at enrollment with partners who found the ring unacceptable were more likely to be nonadherent (aRR 1.55, 95\% CI 1.03, 2.33; $\mathrm{P}=0.04)$, but partner's attitude was not associated with nonadherence among younger participants (aRR 1.02, 95\% CI 0.52, 2.01; $\mathrm{P}=0.96$ ).

\section{Discussion}

Across multiple dimensions of acceptability, the dapivirine vaginal ring was highly acceptable to participants and, from their perspective, was also acceptable to their partners. The majority of participants expressed future likelihood of use. However, components of acceptability varied greatly by country, and certain components of acceptability, like effects on sex, had a significant impact on adherence.

Despite vaginal rings being novel in this population, overall acceptability was high and increased over time as participants gained comfort and familiarity with the ring. These findings are similar to early phase dapivirine ring studies [7-9], and supported by qualitative analysis of ASPIRE data, where participants reported liking the ring more with experience [12]. Without a standardized measurement of acceptability, however, it can be challenging to compare findings across studies. The Mensch model [11] provides a framework to evaluate components of acceptability in the context of clinical trials, which, allowed us to better understand factors that may contribute to overall acceptability and observe that some, but not all, elements of acceptability changed over time. For example, while ease of insertion and general comfort with the ring increased, physically feeling the ring during sex for participants and partners remained relatively stable. By assessing both overall and separate components of acceptability, we gained a deeper understanding of ring acceptability, and demonstrate that even though certain elements of ring use may be less acceptable or may change over time, general acceptability may still be high.

We observed notable differences by country both overall and by individual components of acceptability. Generally, participants in Uganda and Zimbabwe reported higher likelihood of future ring use. Individual elements of acceptability also varied by country. For example, those in Malawi and Uganda minded ring use during sex and during menses, whereas this was not a reported concern among participants from South Africa or Zimbabwe. Furthermore, partner acceptability was much lower in Uganda compared to other countries. It is worth noting that some countryspecific acceptability measures may seem incongruent with each other. For example, participants in Malawi and Uganda reported minding wearing the ring during sex more often but were also more likely to report increased sexual pleasure. In Uganda, future likelihood of use was among the highest, but minding ring use during menses or sex was also high. These findings speak to several key points. First, the various components of acceptability are not necessarily mutually exclusive. For example, one may mind wearing the ring during sex with certain partners (who may not know about or approve of ring use) but feel increased pleasure with other partners. Similarly, one may mind wearing the ring during sex or menses, but desire or need for an effective HIV prevention method-and therefore their intention for future use of an effective product-may outweigh this dislike. Alternatively, some participants may have disregarded the consistent use aspect of the willingness of future use variable ("keep [the ring] inserted in your vagina every day"), which may also explain some of these apparent disparate results-lack of cognitive testing of this question is a limitation of our analysis. Ultimately, participants in the study were likely balancing pleasure, protection, inconvenience, and partnerships simultaneously in their decision-making, as they probably do similarly in many aspects of their lives every day, including but not limited to contraception.

Our findings of variability in acceptability by country are supported by other work, which demonstrate that preferences around sex, product use, vaginal practices, and hygiene, differ across and within countries [17, 18]. Relationship dynamics, previously noted in ASPIRE qualitative research to have varied by location [19], may be one factor that contributes to the observed differences in acceptability by country. Our findings speak to the importance of conducting research across a range of settings and populations, as well as emphasize the need for offering choices in HIV prevention methods. Individuals from different countries, 
cultures and backgrounds have different preferences for HIV prevention products, which has potential to influence their uptake and use.

Given the differences observed in age-stratified efficacy of the dapivirine vaginal ring in ASPIRE [4], we expected to find similar variation in acceptability in younger versus older participants. However, most acceptability measures were similar across ages, apart from younger participants being more likely to report the ring was not acceptable to their primary partner. Although reported partner acceptability varied by age, there was no impact observed on adherence related to this component among younger participants. Younger individuals may be more likely involved in shorter term or more casual relationships, or generally be less comfortable talking to their partners about sexual and reproductive health issues, which could explain lower perceived partner acceptability and/or lack of any observed effect of partner acceptability on adherence in this subpopulation. Our observations may also be limited in that only participants who had access to a ring in the 3 months prior to PUEV were administered acceptability questions-younger participants were less likely to receive a ring during this time, so our findings may underestimate potential effect of partner acceptability on adherence among this group. Nonetheless, these findings highlight that while acceptability may have some influence on adherence, it does not explain age-related differences in observed effectiveness in phase three trials, suggesting that other social and structural factors also contribute to ability to use the ring consistently.

Importantly, although perhaps not unexpectedly, participants with low overall acceptability were less likely to use the ring consistently based on objective adherence measurements. Some attributes of acceptability, including effects on sex, perceived negative change to the vaginal environment, and minding wearing the ring during menses, influenced adherence more strongly, whereas others-comfort and ease of insertion-had no effect. Feeling or minding the ring during sex was the strongest influencer of nonadherence observed at both month 12 and in the last year of the study. This is further supported by our findings of increasing nonadherence among participants who reported no change or decreased sexual pleasure, compared to those who reported increased sexual pleasure with the ring. Our observations are supported by previous qualitative research, which demonstrated that the impact of the ring on sexual experiences was a specific concern that participants felt they had to navigate and that had the potential to impact their adherence [13] and that for some, the ring improved their sexual pleasure for reasons ranging from changes in lubrication to reduced fear of HIV acquisition [12]. Participant report of partner acceptability or their partner feeling the ring during sex had a modest influence on nonadherence, although these results were not statistically significant. A stronger relationship between partner acceptability and adherence was observed among older participants. This trend is supported by other qualitative findings from ASPIRE, where partner perceptions were often cited as a prominent influencer of acceptability and adherence [12] as well as work that looked at the relationship between reported partner support and adherence in ASPIRE [20]. Importantly, perceived partner acceptability does not necessarily equate to partner support, highlighting the importance of exploring these relationships both quantitatively and qualitatively.

Recognizing that acceptability may have some influence on adherence or overall willingness for future use, it is important to acknowledge that actual use (adherence) and intention or willingness to use in the future may not always align. This is especially true when adherence is in the context of a randomized controlled trial (RCT) with a placebo and unknown efficacy of the active product. Future willingness to use a known effective product may be high, even among those who struggled with adherence during the RCT due to challenges with use during sex or other barriers; conversely, a participant with high adherence may not ultimately find a product acceptable. Individuals may have many motivations for joining clinical trials outside of product use, and that these motivations may confound observations between components of acceptability and adherence. Furthermore, other important social and structural factors may also play a role, such as desire to have a child, experience of social harms or intimate partner violence [21], initial worries about the ring [22], perception of HIV risk, or acceptability attributes not measured here, such as product characteristics (size, consistency, smell), dosing regimen, or stigma. That said, understanding what components of acceptability most strongly influence adherence may help identify individuals in need of more support and allow for more targeted counseling during initiation of the ring.

There are a few limitations worth noting in our analysis. First, only participants who received a ring in the past 3 months were asked acceptability questions - meaning that, if those who were lost to follow-up or otherwise not receiving rings (e.g., on product hold) were also more likely to find the ring unacceptable, we may be overestimating acceptability of the ring. However, more than $90 \%$ of participants did complete acceptability questions so we anticipate that acceptability was still high despite this. Furthermore, PUEV ACASI data were not available from two sites in South Africa, where overall acceptability was somewhat lower (available data from South African participants reported only 59\% 'very likely' future use) potentially leading to an underestimate of the strength of association between acceptability and nonadherence. Additionally, we did not evaluate all potential components of acceptability, meaning we could have overlooked other key factors that may or may not influence adherence, or contribute to overall acceptability. 
As already mentioned, we also only measured some acceptability components at PUEV, meaning our analysis of these factors was retrospective and limited our ability to say what effect those factors had on future ring adherence. Also, collection and testing of used rings for residual drug started approximately one year into the trial. Therefore, we are missing some initial residual drug measurements from participants enrolled earlier which could potentially misclassify their level of adherence. Finally, because the error around measures of residual dapivirine with current methods can be up to $0.5 \mathrm{mg}$, it is challenging to define high adherence by a certain release rate (provided that also the amount of dapivirine released per month is small, about $4 \mathrm{mg}$ over 28-days of use) [15]. Hence, we focused on nonadherence as the definition is more definitive. When more granular quantitative measures of adherence for the vaginal ring are developed, we may find a different relationship between acceptability components and high adherence.

In conclusion, the DVR was highly acceptable to participants across several dimensions of acceptability, although with country variation. Acceptability of the ring increased over time, demonstrating the DVR's potential as a prevention product for long-term use. Addressing perceived interference with sex, menses, or problematic changes to the vaginal environment, in future interventions through counseling and skill building could help improve adherence, as could embracing sex-positive messaging related to ring use and increased pleasure. Ultimately, the variability we observed in acceptability of the ring highlight the importance of increased choice in HIV prevention options, as no one method will satisfy the needs of all individuals.

Supplementary Information The online version contains supplementary material available at https://doi.org/10.1007/s10461-021-03205-z.

Acknowledgements The authors would like to acknowledge the full MTN-020/ASPIRE study team and ASPIRE study participants, without which this work would not have been possible. Data management was provided by the Women's Global Health Imperative Program (RTI International, San Francisco, CA) and the Statistical Center for HIV/AIDS Research \& Prevention (Fred Hutchinson Cancer Research Center, Seattle, WA). ACASI build up was provided by Barbara Mensch and Stan Mierzwa (Population Council, New York City, New York). Site laboratory oversight was provided by the Microbicide Trials Network Laboratory Center (Pittsburgh, PA). Study Team Leadership: Jared Baeten, University of Washington (Protocol Chair); Thesla Palanee-Phillips, Wits Reproductive Health and HIV Institute (Protocol Co-chair); Elizabeth Brown, Fred Hutchinson Cancer Research Center (Protocol Statistician); Lydia Soto-Torres, US National Institute of Allergy and Infectious Diseases (Medical Officer); Katie Schwartz, FHI 360 (Clinical Research Manager). Study sites and site Investigators of Record: Malawi, Blantyre site (Johns Hopkins University, Queen Elizabeth Hospital): Bonus Makanani; Malawi, Lilongwe site (University of North Carolina, Chapel Hill): Francis Martinson; South Africa, Cape Town site (University of Cape Town): Linda-Gail Bekker ; South Africa, Durban-Botha's Hill, Chatsworth, Isipingo, Tongaat, Umkomaas, Verulam sites (South African Medical Research Council): Vaneshree Govender, Samantha Siva, Zakir Gaffoor, Logashvari
Naidoo, Arendevi Pather, and Nitesha Jeenarain; South Africa, Durban, eThekwini site (Center for the AIDS Programme for Research in South Africa): Gonasagrie Nair; South Africa, Johannesburg site (Wits RHI): Thesla Palanee-Phillips; Uganda, Kampala site (John Hopkins University, Makerere University): Flavia Matovu; Zimbabwe, Chitungwiza, Seke South and Zengeza sites (University of Zimbabwe College of Health Sciences Clinical Trials Unit): Nyaradzo Mgodi; Zimbabwe, Harare, Spilhaus site (University of Zimbabwe College of Health Sciences Clinical Trials Unit): Felix Mhlanga.

Author Contributions JMB, EB, AVDS, LS-T, TP-P, ETM and AM contributed to the study conception, design, and study operations. TP-P, NJ, LS, KW, IH, KR, TT, PM, TT, MC, and BGM managed site operations, participant enrollment, follow-up, and data collection. Material preparation and data analysis for this manuscript were performed by $\mathrm{EB}$ and $\mathrm{AM}$. The first draft of the manuscript was written by AM and EB and all authors commented on previous versions of the manuscript. All authors read and approved the final manuscript.

Funding The study was designed and implemented by the Microbicide Trials Network (MTN) and funded by the National Institute of Allergy and Infectious Diseases (Grant Nos. UM1AI068633, UM1AI068615, UM1AI106707), with co-funding from the Eunice Kennedy Shriver National Institute of Child Health and Human Development and the National Institute of Mental Health, all components of the U.S. National Institutes of Health (NIH). The content is solely the responsibility of the authors and does not necessarily represent the official views of the National Institutes of Health. The vaginal rings used in this study were supplied by the International Partnership for Microbicides (IPM).

\section{Declarations}

Conflict of Interest The authors have no relevant financial or non-financial interests to disclose.

Ethical Approval All procedures performed in studies involving human participants were in accordance with the ethical standards of the institutional and/or national research committee and with the 1964 Helsinki Declaration and its later amendments or comparable ethical standards. The study was approved by the following Institutional Review Boards/ Ethics Committees and Drug Regulatory Authorities: Prevention Sciences Research Committee of the US National Institute of Allergy and Infectious Diseases; US Food and Drug Administration; National Health Sciences Research Committee of Malawi; Johns Hopkins University Bloomberg School of Public Health Institutional Review Board; University of North Carolina at Chapel Hill Institutional Review Board; Pharmacy, Medicines and Poisons Board of Malawi; University of Cape Town: Human Research Ethics Committee; Biomedical Research Ethics Committee University of KwaZulu-Natal; South African Medical Research Council Ethics Committee; Human Research Ethics Committee: (Medical), University of Witwatersrand, Johannesburg; Medicines Control Council of South Africa; Joint Clinical Research Centre Institutional Review Board; Uganda National Council for Science and Technology; Johns Hopkins University School of Medicine Institutional Review Board; National Drug Authority of Uganda; Medical Research Council of Zimbabwe; Committee on Human Research, University of California-San Francisco; Joint Parirenyatwa Hospital and College of Health Sciences Research Ethics; Research Council of Zimbabwe; Medicines Control Authority of Zimbabwe.

Informed Consent Informed consent was obtained from all individual participants included in the study. 
Open Access This article is licensed under a Creative Commons Attribution 4.0 International License, which permits use, sharing, adaptation, distribution and reproduction in any medium or format, as long as you give appropriate credit to the original author(s) and the source, provide a link to the Creative Commons licence, and indicate if changes were made. The images or other third party material in this article are included in the article's Creative Commons licence, unless indicated otherwise in a credit line to the material. If material is not included in the article's Creative Commons licence and your intended use is not permitted by statutory regulation or exceeds the permitted use, you will need to obtain permission directly from the copyright holder. To view a copy of this licence, visit http://creativecommons.org/licenses/by/4.0/.

\section{References}

1. UNAIDS data: Joint United Nations Programme on HIV/AIDS (UNAIDS). https://www.unaids.org/en/resources/documents/ 2019/2019-UNAIDS-data (2019). Accessed 22 Nov 2020.

2. Shapley-Quinn MK, Manenzhe KN, Agot K, Minnis AM, van der Straten A. "We are not the same": African women's view of multipurpose prevention products in the TRIO clinical study. Int J Womens Health. 2019;11:97-107. https://doi.org/10.2147/IJWH. S185712.

3. Luecke EH, Cheng H, Woeber K, et al. Stated product formulation preferences for HIV pre-exposure prophylaxis among women in the VOICE-D (MTN-003D) study. J Int AIDS Soc. 2016;19(1):20875. https://doi.org/10.7448/IAS.19.1.20875.

4. Baeten JM, Palanee-Phillips T, Brown ER, et al. Use of a vaginal ring containing dapivirine for HIV-1 prevention in women. N Engl J Med. 2016;375(22):2121-32. https://doi.org/10.1056/NEJMo a1506110.

5. Nel A, van Niekerk N, Kapiga S, et al. Safety and efficacy of a dapivirine vaginal ring for HIV prevention in women. N Engl J Med. 2016;375(22):2133-43. https://doi.org/10.1056/NEJMo a1602046.

6. Brown ER, Hendrix CW, van der Straten A, et al. Greater dapivirine release from the dapivirine vaginal ring is correlated with lower risk of HIV-1 acquisition: a secondary analysis from a randomized, placebo-controlled trial. J Int AIDS Soc. 2020;23(11):e25634. https://doi.org/10.1002/jia2.25634.

7. Nel A, Bekker LG, Bukusi E, et al. Safety, acceptability and adherence of dapivirine vaginal ring in a microbicide clinical trial conducted in multiple countries in sub-Saharan Africa. PLoS ONE. 2016;11(3):e0147743. https://doi.org/10.1371/journal.pone. 0147743.

8. Montgomery ET, van der Straten A, Cheng H, et al. Vaginal ring adherence in sub-Saharan Africa: expulsion, removal, and perfect use. AIDS Behav. 2012;16(7):1787-98. https://doi.org/10.1007/ s10461-012-0248-4.

9. van der Straten A, Montgomery ET, Cheng H, et al. High acceptability of a vaginal ring intended as a microbicide delivery method for HIV prevention in African women. AIDS Behav. 2012;16(7):1775-86. https://doi.org/10.1007/s10461-012-0215-0.

10. van der Straten A, Panther L, Laborde N, et al. Adherence and acceptability of a multidrug vaginal ring for HIV prevention in a phase I study in the United States. AIDS Behav. 2016;20(11):2644-53. https://doi.org/10.1007/ s10461-016-1299-8.

11. Mensch BS, van der Straten A, Katzen LL. Acceptability in microbicide and PrEP trials: current status and a reconceptualization.
Curr Opin HIV AIDS. 2012;7(6):534-41. https://doi.org/10.1097/ COH.0b013e3283590632.

12. Montgomery ET, van der Straten A, Chitukuta M, et al. Acceptability and use of a dapivirine vaginal ring in a phase III trial [published correction appears in AIDS 2017 Nov 28;31(18):2563]. AIDS. 2017;31(8):1159-67. https://doi.org/10.1097/QAD.00000 00000001452

13. Laborde ND, Pleasants E, Reddy K, et al. Impact of the dapivirine vaginal ring on sexual experiences and intimate partnerships of women in an HIV prevention clinical trial: managing ring detection and hot sex. AIDS Behav. 2018;22(2):437-46. https://doi.org/ 10.1007/s10461-017-1977-1.

14. Husnik MJ, Brown ER, Marzinke M, et al. Implementation of a novel adherence monitoring strategy in a phase III, blinded, placebo-controlled, HIV-1 prevention clinical trial. J Acquir Immune Defic Syndr. 2017;76(3):330-7. https://doi.org/10.1097/ QAI.0000000000001503.

15. Spence P, Nel A, van Niekerk N, Derrick T, Wilder S, Devlin B. Post-use assay of vaginal rings (VRs) as a potential measure of clinical trial adherence. J Pharm Biomed Anal. 2016;125:94-100. https://doi.org/10.1016/j.jpba.2016.03.023.

16. Nel A, Haazen W, Nuttall J, Romano J, Rosenberg Z, van Niekerk N. A safety and pharmacokinetic trial assessing delivery of dapivirine from a vaginal ring in healthy women. AIDS. 2014;28(10):1479-87. https://doi.org/10.1097/QAD.0000000000 000280 .

17. Montgomery ET, Beksinska M, Mgodi N, et al. End-user preference for and choice of four vaginally delivered HIV prevention methods among young women in South Africa and Zimbabwe: the Quatro Clinical Crossover Study. J Int AIDS Soc. 2019;22(5):e25283. https://doi.org/10.1002/jia2.25283.

18. Minnis AM, Roberts ST, Agot K, et al. Young women's ratings of three placebo multipurpose prevention technologies for HIV and pregnancy prevention in a randomized, cross-over study in Kenya and South Africa. AIDS Behav. 2018;22(8):2662-73. https://doi. org/10.1007/s10461-018-2078-5.

19. Pleasants E, Tauya T, Reddy K, et al. Relationship type and use of the vaginal ring for HIV-1 prevention in the MTN 020/ASPIRE trial. AIDS Behav. 2020;24(3):866-80. https://doi.org/10.1007/ s10461-019-02521-9.

20. Roberts ST, Nair G, Baeten JM, et al. Impact of male partner involvement on women's adherence to the dapivirine vaginal ring during a phase III HIV prevention trial. AIDS Behav. 2020;24(5):1432-42. https://doi.org/10.1007/ s10461-019-02707-1.

21. Palanee-Phillips T, Roberts ST, Reddy K, et al. Impact of partnerrelated social harms on women's adherence to the dapivirine vaginal ring during a phase III trial. J Acquir Immune Defic Syndr. 2018;79(5):580-9. https://doi.org/10.1097/QAI.0000000000 001866 .

22. van der Straten A, Browne EN, Shapley-Quinn MK, et al. First impressions matter: how initial worries influence adherence to the dapivirine vaginal ring. J Acquir Immune Defic Syndr. 2019;81(3):304-10. https://doi.org/10.1097/QAI.0000000000 002028 .

Publisher's Note Springer Nature remains neutral with regard to jurisdictional claims in published maps and institutional affiliations. 